\title{
Consumo de dietas "low carb high fat high protein" suas implicações metabólicas, clínicas e nutricionais: uma revisão integrativa
}

Consumption of low carb high fat high protein diets and its metabolic, clinical and nutritional implications: an integrative review

Consumo de dietas bajas en carbohidratos alta en grasas alta en proteína y sus implicaciones metabólicas, clínicas y nutricionales: una revisión integrativa

Saulo Leite de Paula

ORCID: https://orcid.org/0000-0002-6511-6115 Universidade Federal do Ceará, Brasil E-mail: saulo.paula@ebserh.gov.br

Maria Rosimar Teixeira Matos ORCID: https://orcid.org/0000-0002-4239-3151 Universidade Estadual do Ceará, Brasil E-mail: rosimar.matos@uece.br

Yanna Cavalcante Martins ORCID: https://orcid.org/0000-0002-1745-0847 Faculdade Mauricio de Nassau, Brasil

E-mail: Cavalcantedepaulay@gmail.com

Noênia Alves de Araújo

ORCID: https://orcid.org/0000-0002-4048-4199 Universidade Estadual do Ceará, Brasil

E-mail: noenianutri@gmail.com

Francisco Sydney Henrique da Silva ORCID: https://orcid.org/0000-0002-4771-6570

Universidade Estadual do Ceará, Brasil

E-mail: sidneyhenrique.08@hotmail.com

Mikaelle Alves Silva

ORCID: https://orcid.org/0000-0002-9336-1491 Universidade Federal do Espírito Santo, Brasil E-mail: farm.mikaelle@gmail.com

Kleber Luiz Santana dos Santos ORCID: https://orcid.org/0000-0003-1781-6391 Universidade Unicesumar, Brasil E-mail: santanared@gmail.com

Gerson Pedroso de Oliveira ORCID: https://orcid.org/0000-0002-3532-887X Centro de Pós Graduação e Aperfeiçoamento LTDA, Brasil E-mail: gerson-06@hotmail.com

Weberton Dorásio Sobrinho

ORCID: https://orcid.org/0000-0003-0619-7214 Universidade de Rio Verde, Brasil

E-mail: dorasioweberton@gmail.com

Thalita Rayanne da Silva Silva ORCID: https://orcid.org/0000-0003-2239-8649

Faculdade Santa Terezinha, Brasil

E-mail: silvathalitarayanne@gmail.com

Natasha Cristina Rangel Rodrigues ORCID: https://orcid.org/0000-0001-7441-9136

Universidade Federal do Pará, Brasil

E-mail: natasharange194@ hotmail.com

Leandro Barbosa Teixeira

ORCID: https://orcid.org/0000-0002-9444-7828

Universidade Estácio de Sá, Brasil

E-mail: Lbtteixeira@ hotmail.com

Joycianne Ramos Vasconcelos de Aguiar ORCID: https://orcid.org/0000-0002-0203-4939 Heufpel, Brasil

E-mail: joycianneaguiar@hotmail.com 


\title{
Resumo
}

O consumo da dieta da "moda" “Low Carb High fat High Protein' (LCHFHP) está cada vez mais frequente na atualidade por ocasionar perda de peso mais rápida e consequente melhoria na qualidade de vida. A presente revisão integrativa visa identificar as implicações metabólicas, clínicas e nutricionais relacionadas ao consumo de dietas Low Carb High fat High Protein. A pesquisa bibliográfica foi realizada através de buscas nas bases de dados eletrônicas Pubmed Central e Elsevier, no período de 2006 a 2018, com a utilização dos seguintes descritores: "carbohydrate; dietary carbohydrates; diet, low carbohydrate; diet, carbohydrate-restricted; ketosis; diabetes; motor active; obesity; cardiovascular disease; protein; physical activite.” Inicialmente foram encontrados 68.219 artigos. Desses, 40 foram pré-selecionados cumprindo os critérios de inclusão. Porém, dentre estes 40, 28 estavam dentro do perfil do estudo. A maioria dos estudos embora demonstrando efeitos positivos com o consumo de dieta LCHFHP, ao curto prazo, quanto à perda de peso, redução no percentual de gordura corporal, melhora da sensibilidade à insulina e no controle glicêmico e redução na utilização de medicações, mostraram que nem sempre esses efeitos foram atribuídos à redução de carboidratos na dieta. Além desses benefícios não terem sido evidenciados ao longo prazo, observou-se aumento nos níveis séricos dos biomarcadores inflamatórios, da extensão da aterosclerose e na morbidade e mortalidade cardiovascular. Realizou-se de mais estudos, principalmente ao longo prazo e com amostragem maior, no sentido de melhor averiguar os benefícios ou riscos decorrentes do consumo dessas dietas, bem como mecanismo de ação, principalmente diante das doenças crônicas não transmissíveis.

Palavras-chaves: Dieta restrita em carboidratos; Composição corporal; Obesidade; Diabetes mellitus; Doenças cardiovasculares.

\begin{abstract}
The consumption of the "fad" diet "Low Carb High fat High Protein" (LCHFHP) is increasingly frequent nowadays because it causes faster weight loss and consequent improvement in quality of life. This integrative review aims to identify the metabolic, clinical and nutritional implications related to the consumption of Low Carb High fat High Protein diets. The bibliographic research was carried out through searches in the electronic databases Pubmed Central and Elsevier, from 2006 to 2018, using the following descriptors: "carbohydrate; dietary carbohydrates; diet, low carbohydrate; diet, carbohydrate-restricted; ketosis; diabetes; active engine; obesity; cardiovascular disease; protein; physical activity." Initially, 68,219 articles were found. Of these, 40 were pre-selected fulfilling the inclusion criteria. However, among these 40, 28 were within the study profile. Most studies, although demonstrating positive effects with the consumption of the LCHFHP diet, in the short term, in terms of weight loss, reduction in the percentage of body fat, improvement in insulin sensitivity and glycemic control and reduction in the use of medications, showed that these effects were not always attributed to the reduction of carbohydrates in the diet. In addition to these long-term benefits, there was an increase in serum levels of inflammatory biomarkers, in the extent of atherosclerosis and in cardiovascular morbidity and mortality. More studies were carried out, mainly over the long term and with a larger
\end{abstract}


sample, in order to better investigate the benefits or risks arising from the consumption of these diets, as well as the mechanism of action, especially in the face of non-communicable chronic diseases.

Keywords: Diet, Carbohydrate-Restricted; Body Composition; Obesity; Diabetes mellitus; Cardiovascular disease.

\begin{abstract}
Resumen
El consumo de la dieta "de moda" "Low Carb High fat High Protein" (LCHFHP) es cada vez más frecuente en la actualidad porque provoca una pérdida de peso más rápida y la consecuente mejora en la calidad de vida. Esta revisión integradora tiene como objetivo identificar las implicaciones metabólicas, clínicas y nutricionales relacionadas con el consumo de dietas bajas en carbohidratos, ricas en grasas y ricas en proteínas. La búsqueda bibliográfica se realizó mediante búsquedas en las bases de datos electrónicas Pubmed Central y Elsevier, de 2006 a 2018, utilizando los siguientes descriptores: "carbohidrato; carbohidratos dietéticos; dieta baja en carbohidratos; dieta restringida en carbohidratos; cetosis; diabetes; motor activo; obesidad; enfermedad cardiovascular; proteína; actividad física." Inicialmente se encontraron 68.219 artículos. De estos, 40 fueron preseleccionados cumpliendo los criterios de inclusión. Sin embargo, entre estos 40, 28 estaban dentro del perfil de estudio. La mayoría de los estudios, aunque demostraron efectos positivos con el consumo de la dieta LCHFHP, a corto plazo, en términos de pérdida de peso, reducción del porcentaje de grasa corporal, mejora de la sensibilidad a la insulina y control glucémico y reducción del uso de medicamentos, mostraron que estos efectos no siempre se atribuyeron a la reducción de carbohidratos en la dieta. Además de estos beneficios a largo plazo, hubo un aumento en los niveles séricos de biomarcadores inflamatorios, en la extensión de la aterosclerosis y en la morbilidad y mortalidad cardiovascular. Se realizaron más estudios, principalmente a largo plazo y con una muestra más amplia, con el fin de investigar mejor los beneficios o riesgos derivados del consumo de estas dietas, así como el mecanismo de acción, especialmente frente a enfermedades no transmisibles. enfermedades crónicas.
\end{abstract}

Palabras clave: Dieta restringida en carbohidratos; Composición corporal; Obesidad; Diabetes mellitus; Enfermedad cardiovascular.

\title{
1. Introdução
}

A obesidade tem se tornado uma pandemia mundial e é um dos principais fatores de risco para desenvolvimento de diabetes mellitus tipo 2 e doença cardiovascular, existindo poucas opções tangíveis e seguras para o tratamento da mesma (Sansbury \& Hill, 2014).

Segundo Ghroubi et al. (2009), o tratamento da obesidade está relacionado com a combinação de atividade física, medidas dietéticas e psicológicas. Nos seus estudos foram observadas mudanças quanto a perda de peso, diminuição do percentual de gordura, redução da circunferência abdominal, aumento da força muscular, aumento da capacidade aeróbia e correção dos distúrbios metabólicos.

A principal causa desta doença está relacionada com o desequilíbrio energético, explicado pelo consumo de calorias maior que do que a utilização das mesmas; entretanto outras causas de difícil manipulação ou modificação, como por exemplo, ocupação, ambiente estrutural e influências sociais estão relacionadas com esta condição, de forma que os alvos mais viáveis para a terapia são os processos moleculares e vias que regulam o metabolismo energético ou ingestão calórica (Sansbury \& Hill, 2014).

Segundo Busetto, Marangon \& Stefano (2011), a maior parte dos comitês internacionais e organizações científicas sugerem a utilização de uma dieta moderadamente baixa quanto ao valor calórico, com baixo teor de gordura (principalmente da porção saturada).

Nas dietas de redução de peso, a importância da composição dos macronutrientes tem sido motivo de uma série de estudos. Apesar de serem indicadas dietas com restrição calórica e redução na fração lipídica, efeitos positivos para esta condição foram observados com o consumo de dietas com baixa quantidade de carboidratos e elevadas frações proteicas (Stocks et al., 2007).

Com o avanço epidêmico da obesidade e de acordo com as preocupações implicadas nas suas complicações, intervenções diferenciadas, como as dietas de baixo consumo de carboidrato, têm sido utilizadas e investigadas, dietas com restrição de carboidratos, como a Atkins New Diet Revolution, relacionam-se com uma ingestão de proteínas e gorduras de 
forma liberal e uma restrição, muitas vezes severa, na ingestão de carboidratos (Coleman \& Nickols-Richardson, 2005). Uma série de mecanismos tem sido proposta na tentativa de explicar a possível superioridade das dietas de baixo teor de carboidrato frente às dietas convencionais de baixa gordura (Buseto, Marago \& de Stefno, 2011).

Hipóteses inovadoras tentaram vincular às adaptações bioquímicas as mudanças sistemáticas e metabólicas induzidas pelas dietas com baixos teores de carboidratos. Apesar disso, não se teve clareza quanto e quais dos mecanismos potenciais propostos são diretamente relevantes aos efeitos clínicos desta dieta. Entretanto, é pouco provável a existência de um único mecanismo ou de uma via final comum em unificar as numerosas hipóteses (Rho \& Sankar, 2008).

No entanto, a grande maioria das sociedades científicas de nutrição mostrou a posição às dietas baseadas em baixo consumo de carboidratos. Devido à ingestão comprometida de vitaminas e minerais e potencial risco de lesões renais, ósseas, hepáticas e cardíacas, que podem se agravar de acordo com o tipo de proteína (origem vegetal ou animal) e de carboidrato ( simples ou complexo) inseridos, estas recomendaram a inserção de mais carboidratos complexos e menos gorduras na dieta Kim et al.,2008 \& Nilsson et al., 2012).

Portanto, diante da crescente utilização das dietas de "moda" como a dieta Low Carb High Fat High Protein (baixo consumo de carboidratos e alto consumo de gorduras e proteína) no controle do sobrepeso e obesidade e mudança da composição corporal, e das divergências quanto à sua indicação por suas implicações metabólicas, clínicas e nutricionais, assim objetivou-se identificar essas implicações decorrentes do consumo desta dieta.

\section{Metodologia}

Estudo descritivo, realizado através de levantamento bibliográfico por método de revisão integrativa, sobre o consumo de dietas Low Carb High Fat High Protein e suas implicações metabólicas, clínicas e nutricionais.

Procedeu-se a busca de artigos científicos publicados nos últimos 12 anos (2006 a 2018), através das bases de dados eletrônicas Science Direct (ELSEVIER) e Pubmed Central (PMC). A seleção dos descritores utilizados no processo de revisão foi efetuada mediante consulta ao DeCS (descritores de assunto em ciências da saúde da biblioteca virtual em saúde - BVS) e também da utilização de palavras-chave.

Foram utilizados os seguintes descritores: "carbohydrate; dietary carbohydrates; diet, low carbohydrate; diet, carbohydrate-restricted; ketosis; diabetes; motor active; obesity; cardiovascular disease; protein; physical activite." As buscas foram feitas, quando necessário, através de combinações entre os mesmos considerando o operador booleano representado pelo termo "AND”, dependendo da base de dados pesquisada. Nas estratégias de busca nas bases de dados foram consideradas as seguintes combinações: physical activite AND low carbohydrate high protein; low carbohydrate high protein diet AND obesity; low carbohydrate diet AND cardiovascular diabetes; low carbohydrate diet AND cardiovascular disease.

Foram incluídos estudos de artigo original, de caráter observacional e intervencionista e de delineamento longitudinal e transversal, publicados na íntegra entre os anos de 2006 e 2018. Foram excluídas dissertações, teses, monografias, publicações de anais de congressos e artigos de revisão.

O fluxograma mostra a distribuição dos artigos encontrados e selecionados por base de dados. Foram encontrados nas duas bases de dados um total de 68219 artigos, destes quarenta foram pré-selecionados e vinte e oito analisados. 
Research, Society and Development, v. 10, n. 16, e282101623575, 2021

(CC BY 4.0) | ISSN 2525-3409 | DOI: http://dx.doi.org/10.33448/rsd-v10i16.23575

Fluxograma - Distribuição dos artigos encontrados e selecionados por base de dados.

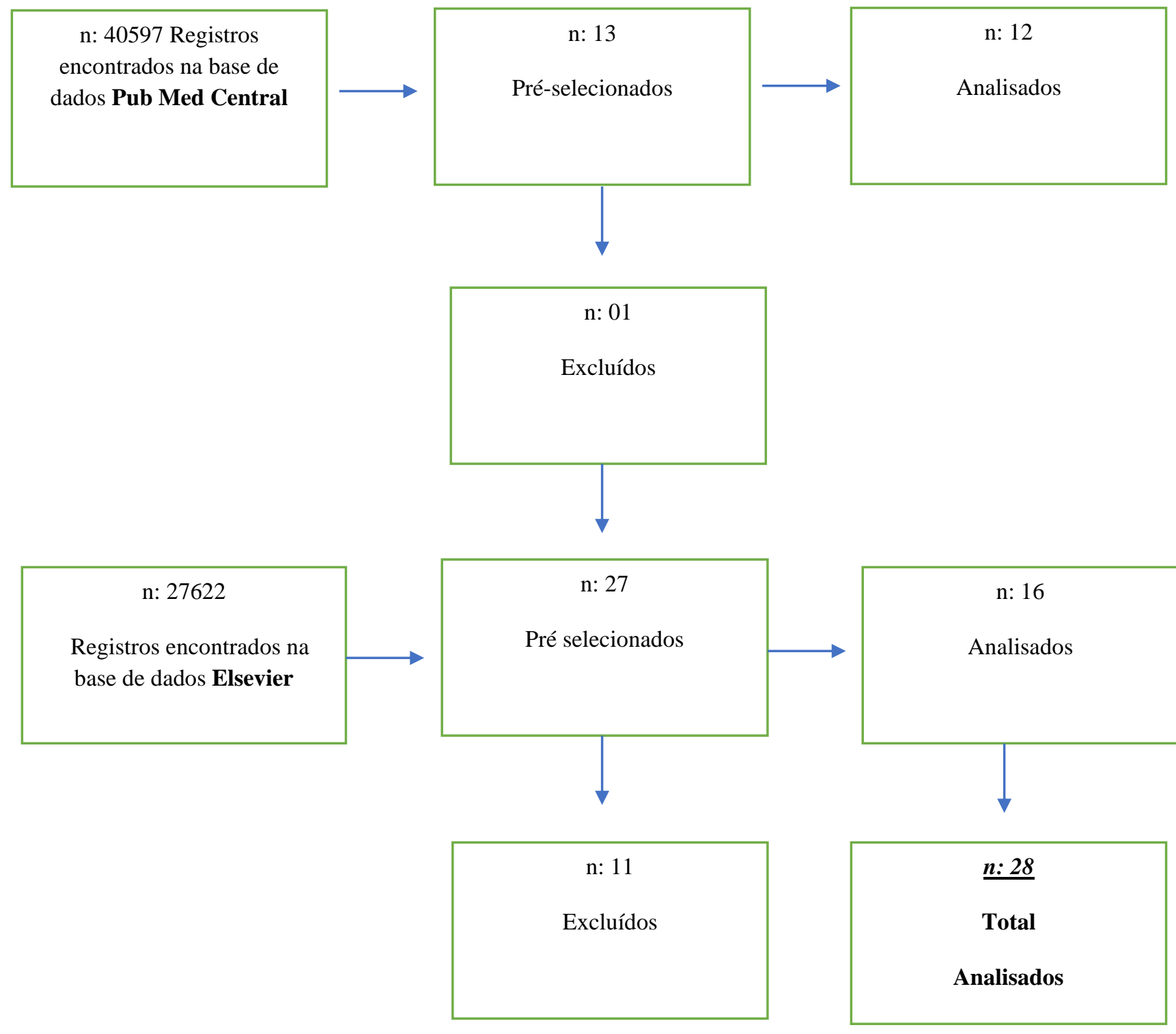

Fonte: Produzido pelos autores (2021).

Os artigos incluídos foram agrupados e posteriormente analisados em três blocos de acordo com as abordagens mais evidenciadas acerca das implicações metabólicas e clínicas e nutricionais.

\section{Resultados e Discussão}

Os resultados e discussão serão apresentados em tópicos, conforme as abordagens mais evidenciadas descritas na metodologia. 


\section{Consumo de Dieta Low Carb High Fat High Protein na Obesidade}

A Tabela 1 mostra uma síntese (autoria, ano de publicação, tipo de intervenção e resultados encontrados) dos estudos que investigaram a influência do consumo da dieta LCHFHP na perda de peso e mudança de composição corporal na população obesa avaliada.

Tabela 1 - Síntese dos estudos que investigaram a influência do consumo da dieta Low Carb High Fat High Protein na perda de peso e mudança de composição corporal.

\begin{tabular}{|c|c|c|}
\hline Autoria/Ano & Intervenção & Resultados \\
\hline Kevin et al. (2015) & $\begin{array}{l}\text { Estudo experimental randomizado/ } 10 \\
\text { indivíduos do sexo masculino e } 9 \text { do sexo } \\
\text { feminino com obesidade/ acompanhados por } \\
02 \text { semanas. }\end{array}$ & $\begin{array}{l}\downarrow \text { secreção de insulina, } \uparrow \text { oxidação de gorduras e } \uparrow \\
\text { perda de gordura corporal, em comparação com } \\
\text { uma dieta de base eucalórica. Entretanto, em } \\
\text { quantidades isocalóricas, observou-se que } \downarrow \text { de } \\
\text { gordura na dieta levou a uma maior } \downarrow \text { de gordura } \\
\text { corporal do que a restrição de carboidratos na dieta } \\
\text { em adultos com obesidade. }\end{array}$ \\
\hline Nancy et al. (2010) & $\begin{array}{l}\text { Estudo experimental randomizado/ } 46 \\
\text { sujeitos (33 completaram a intervenção) / } \\
\text { acompanhados por } 12 \text { semanas }\end{array}$ & $\begin{array}{l}\downarrow \text { IMC-Z-score (IMC-Z) significativamente } \uparrow \text { para o } \\
\text { grupo HPLC. Dieta HPLC é uma opção segura e } \\
\text { eficaz para perda de peso supervisionada por } \\
\text { médicos em adolescentes com obesidade grave. }\end{array}$ \\
\hline Jeannie et al. (2008) & $\begin{array}{l}\text { Estudo experimental randomizado/ } 88 \text { adultos } \\
\text { obesos abdominais/ acompanhados por } 24 \\
\text { semanas }\end{array}$ & $\begin{array}{l}\text { Em condições isocalóricas, as dietas VLCHF e } \\
\text { HCLF resultaram em perda de peso similar. }\end{array}$ \\
\hline Jian \& Steven (2013) & $\begin{array}{l}\text { Estudo experimental randomizado/ } 250 \text { ratos } \\
\text { machos/ acompanhados por } 7 \text { semanas }\end{array}$ & $\begin{array}{l}\text { Dieta com alto teor de gordura e baixo teor de } \\
\text { carboidrato resultou em } \uparrow \text { ganho de peso. }\end{array}$ \\
\hline De Luiz et al. (2015) & $\begin{array}{l}\text { Estudo experimental randomizado/ } 331 \\
\text { indivíduos obesos/ acompanhados por } 09 \\
\text { meses }\end{array}$ & $\begin{array}{l}\text { Com as dietas HP e } S \text {, observaram-se redução do } \\
\text { IMC, peso, massa gorda, circunferência da cintura e } \\
\text { na relação cintura-quadril. Foi observado que uma } \\
\text { dieta hipocalórica rica em proteínas (HP) / baixo } \\
\text { carboidrato apresenta uma } \uparrow \text { perda de peso que uma } \\
\text { dieta hipocalórica padrão em proteínas (S). }\end{array}$ \\
\hline Tanja et al. (2013) & $\begin{array}{l}\text { Estudo experimental randomizado/ } 771 \\
\text { adultos obesos ( } 585 \text { completaram o estudo) / } \\
\text { acompanhados por } 10 \text { semanas }\end{array}$ & $\begin{array}{l}\text { Dieta com } \downarrow \text { teor de gordura com } \uparrow \% \text { de proteína } \\
(+2 \%) \text { houve perda de } 1,1 \mathrm{~kg} \text { a mais que o grupo } \\
\text { com dieta hiperlipídica com redução do } \% \text { de } \\
\text { proteína }(-2 \%) \text { ao longo de } 10 \text { semanas. }\end{array}$ \\
\hline Johnstone et al. (2011) & $\begin{array}{lll}\text { Estudo experimental randomizado/ } & 16 \\
\text { homens obesos/ acompanhados por } 4 \\
\text { semanas }\end{array}$ & $\begin{array}{l}\text { Em média, os sujeitos perderam } 6,75 \text { e } 4,32 \mathrm{~kg} \text { de } \\
\text { peso nas dietas LC (baixo carboidrato) e MC } \\
\text { (médio carboidrato), respectivamente. Dietas } \uparrow \text { alto } \\
\text { teor de proteína para perda de peso (MC WL) são } \\
\text { seguras dentro deste prazo relativamente curto } \\
\text { período de tempo ( } 04 \text { semanas). }\end{array}$ \\
\hline Cherrie et al. (2007) & $\begin{array}{l}\text { Estudo randomizado/ } 25 \text { mulheres com } \\
\text { sobrepeso/ acompanhados por } 16 \text { semanas }\end{array}$ & $\begin{array}{l}\text { Devido ao } \uparrow \text { de sentimento de bem-estar, é possível } \\
\text { que as dietas HPLC sejam associadas com melhor } \\
\text { adesão e, portanto, mais bem sucedidas no } \\
\text { tratamento a longo prazo da obesidade. }\end{array}$ \\
\hline Stijn et al. (2012) & $\begin{array}{l}\text { Estudo experimental randomizado/ } 132 \\
\text { indivíduos/ acompanhados por } 12 \text { meses }\end{array}$ & $\begin{array}{l}\text { A perda de peso corporal e a manutenção do peso } \\
\text { dependem do alto teor de proteína, mas não do } \\
\text { "low-carb" componente da dieta. }\end{array}$ \\
\hline
\end{tabular}

Fonte: Produzido pelos autores (2021).

Dos vinte e oito artigos reunidos nesta revisão, nove (32\%) investigaram a influência do consumo dessas dietas na perda de peso e mudança na composição corporal de pacientes obesos. Dentre esses, observou-se que oito (88,88\%) das intervenções mostraram tais efeitos, porém nem sempre atribuídos à redução de carboidratos. 
Em apenas um estudo, Jian \& Steven (2013), não se verificou esses efeitos com a utilização desse tipo de dieta. Por outro lado, Kevin et al. (2015) mostrou que apesar de melhor perda de peso em relação a uma dieta eucalórica, em quantidades isocalóricas, a redução do lipídio da dieta foi mais eficaz na perda de peso do que a redução de carboidrato. Dois outros estudos, realizados por De Luiz et al. (2015) \& Stijn et al. (2012), mostraram que apesar da diminuição de peso com dietas LCHFHP, essa perda foi mais efetiva devido ao alto teor de proteína que o baixo teor de carboidrato; isso porque o consumo elevado de proteína reduziu o apetite, com subsequente consumo menor de energia, e aumentou o efeito termogênico dos alimentos. Resultado corroborado no estudo de Tanja et al. (2013), em que a dieta com baixo teor de lipídio e alto teor de proteína resultou em maior perda de peso que uma dieta com alto teor de gordura e reduzida em proteína.

Na meta-análise de Meng et al. (2017), na revisão sistemática de Hu \& Bazzano (2013) e na pesquisa de Jeannie et al. (2008), em discordância aos demais estudos analisados, não se observaram diminuição de peso corporal.

Convém ressaltar, que todos os estudos analisados foram experimentais randomizados e realizados com seres humanos, com exceção o de Jian \& Steven (2013) que utilizaram ratos na sua amostra, porém todos foram realizados em um período inferior a seis meses, com exceção das pesquisas de Jeannie et al.(2008), De Luiz et al.(2015) \& Stijn et al. (2012).

Dentre os pontos fortes verificados nesses estudos destacam-se o fato de terem sido realizados com seres humanos, em sua maioria; o consumo de dietas isocalóricas, com variação na distribuição dos macronutrientes; o pareamento de indivíduos pelo peso corporal, idade e IMC; a utilização da absortometria radiológica de dupla energia (DEXA) na determinação da gordura corporal antes e depois do estudo; a presença de alguns critérios de exclusão como: diabetes mellitus tipo 2; doenças hepáticas ou renais, entre outros, que poderiam interferir nos resultados; a exclusão de medicações, que impactariam no apetite; e a coleta das informações detalhadas sobre suas dietas, no início e durante o acompanhamento, pelo nutricionista.

Dentre os pontos fracos verificados nas pesquisas avaliadas, ressaltam-se o tempo de acompanhamento a curto prazo; amostras pequenas; utilização de fórmulas preditivas para estimar gasto energético de repouso; altas taxas de desistência; dificuldade de adesão à dieta; ausência de grupo controle; população mais jovem e com peso normal ao invés de mais idosa e obesa; amostra constituída por um sexo; falta de fatores de confusão; variação do número de dias de registro alimentar entre os sujeitos; utilização de escores subjetivos de fome e saciedade nas escalas analógicas visuais, sem medições de marcadores biológicos relacionados ao apetite e saciedade (como por exemplo, grelina \& leptina).

\section{Consumo de Dieta Low Carb High Fat High Protein no Controle do Diabetes Mellitus Tipo 2}

A Tabela 2 mostra uma síntese (autoria, ano de publicação, tipo de intervenção e resultados encontrados) dos estudos que investigaram a influência do consumo da dieta LCHFHP no controle do diabetes mellitus tipo 2 na população investigada. 
Tabela 2 - Síntese dos estudos que investigaram a influência do consumo da dieta Low Carb High Fat High Protein no controle do diabetes mellitus tipo 2.

\begin{tabular}{|c|c|c|}
\hline Autores/Ano & Método & Resultados \\
\hline Keiko et al. (2014) & $\begin{array}{l}\text { Estudo experimental randomizado/18 ratos } \\
\text { obesos e diabéticos induzidos/acompanhados } \\
\text { por16 semanas }\end{array}$ & $\begin{array}{l}\text { Glicemia de jejum e de IRI em jejum foram } \\
\text { significativamente mais } \uparrow \text { em dieta com } \\
\text { restrição severa de carboidratos que em dieta } \\
\text { padrão. Intolerância à glicose, } \downarrow \text { níveis de } \\
\text { FGF21 e } \downarrow \text { expressão de SCD1 hepática } \\
\text { indicaram que uma dieta baixa em carboidratos } \\
\text { leva a manifestações metabólicas deletérias. }\end{array}$ \\
\hline Westman et al. (2008) & $\begin{array}{l}\text { Estudo experimental randomizado/ } 84 \\
\text { indivíduos obesos com DM2 / acompanhados } \\
\text { por } 24 \text { semanas/ }\end{array}$ & $\begin{array}{l}\text { Melhorias na hemoglobina A1c, glicemia de } \\
\text { jejum, insulina em jejum e perda de peso. } \\
\text { Medicamentos para DM2 foram reduzidos ou } \\
\text { eliminados em } 95,2 \% \text { na dieta cetogênica com } \\
\text { baixo teor de carboidratos (LCKD) vs. } 62 \% \text { dos } \\
\text { participantes dieta com baixo índice glicêmico } \\
\text { (LGID). }\end{array}$ \\
\hline Saslow et al. (2014) & $\begin{array}{l}\text { Estudo experimental randomizado/ } 34 \\
\text { indivíduos com excesso de peso ou obesos com } \\
\text { DM2 ou pré-diabetes/ Acompanhados por } 03 \\
\text { meses }\end{array}$ & $\begin{array}{l}\text { Nível médio de HbA1c: manteve-se inalterado } \\
\text { no grupo de dieta com carboidratos em } \\
\text { quantidade média, } \downarrow \text { gordura, restrita em } \\
\text { calorias e contagem de carboidratos (MCCR). } \downarrow \\
0,6 \% \text { no grupo dieta com } \downarrow \text { teor de carboidratos, } \\
\uparrow \text { gordura e restrição de calórica (LCK). } \\
\text { Interrupção de um ou mais medicamentos para } \\
\text { DM2: } 44 \% \text { do grupo LCK e } 11 \% \text { do grupo } \\
\text { MCCR (p = } 0,03 \text { ); } 31 \% \text { descontinuaram as } \\
\text { sulfonilureias no grupo LCK. }\end{array}$ \\
\hline Lawrence et al. (2011) & $\begin{array}{l}\text { Estudo de coorte prospectivo/ } 40.475 \\
\text { indivíduos sem DM2, doença cardiovascular ou } \\
\text { câncer no início do estudo/ acompanhados por } \\
\text { cerca de } 20 \text { anos }\end{array}$ & $\begin{array}{l}\text { Consumo de proteína vegetal e elevado em } \\
\text { gordura não foi significativamente associado ao } \\
\text { risco de DM2 global, mas foi inversamente } \\
\text { associado à DM2 em homens com } 65 \text { anos de } \\
\text { idade. Dieta pobre em carboidratos e alta em } \\
\text { proteína e gordura animal foi positivamente } \\
\text { associada ao risco de DM2 em homens. Dietas } \\
\text { com baixo teor de carboidratos devem obter } \\
\text { proteína e gordura de alimentos que não sejam } \\
\text { provenientes de carne vermelha e processada. }\end{array}$ \\
\hline Alaa et al. (2009) & $\begin{array}{l}\text { Estudo experimental randomizado/ } 63 \text { ratos } \\
\text { machos diabéticos induzidos/ acompanhados } \\
\text { por } 08 \text { semanas }\end{array}$ & $\begin{array}{l}\text { Dieta cetogênica com baixo teor de carboidratos } \\
\text { (LCKD) foi eficaz em trazer o nível de glicose } \\
\text { no sangue próximo ao normal indicando efeito } \\
\text { significativo na melhoria do estado diabético e } \\
\text { ajudando a estabilizar a hiperglicemia. }\end{array}$ \\
\hline Goldsteinet al. (2011) & 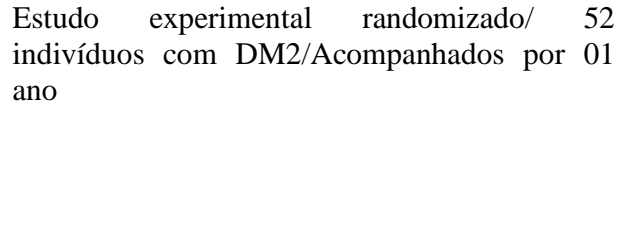 & $\begin{array}{l}\text { Perda de peso e diminuição na HbAlc foram } \\
\text { semelhantes em ambos os grupos. Melhoria no } \\
\text { controle glicêmico acompanhou a modesta } \\
\text { redução de peso, não havendo vantagem } \\
\text { significativa em termos de controle glicêmico } \\
\text { com a dieta tipo Atkins, que teve menor adesão. }\end{array}$ \\
\hline Von et al. (2013) & $\begin{array}{l}\text { Estudo experimental randomizado/ } 16 \\
\text { indivíduos com DM2, sem doença cardíaca/ } \\
\text { acompanhados por } 03 \text { semanas }\end{array}$ & $\begin{array}{l}\text { Nos grupos paralelos, ambas as dietas } \\
\text { induziram } \downarrow \text { similares de HbA1c, dieta de baixo } \\
\text { carboidrato (LC) melhorou consideravelmente a } \\
\text { resistência à insulina. Proinsulina intacta pós- } \\
\text { prandial permaneceu inalterada na dieta de } \\
\text { baixa gordura (LF), mas diminuiu com LC } \\
\text { subsequente. Dieta com baixo índice glicêmico; } \\
\text { alto teor de proteína, mas não um baixo teor de } \\
\text { gordura; e alto teor de carboidrato, melhora a } \\
\text { resistência à insulina e pode prevenir ou } \\
\text { retardar o início da cardiomiopatia diabética. }\end{array}$ \\
\hline
\end{tabular}


Research, Society and Development, v. 10, n. 16, e282101623575, 2021

(CC BY 4.0) | ISSN 2525-3409 | DOI: http://dx.doi.org/10.33448/rsd-v10i16.23575

\begin{tabular}{|l|l|l|}
\hline Jackson et al. (2010) & $\begin{array}{l}\text { Estudo experimental randomizado/ 06 homens } \\
\text { saudáveis e fisicamente ativos }\end{array}$ & $\begin{array}{l}\text { A glicemia de jejum foi significativamente } \\
\text { reduzida. }\end{array}$ \\
\hline Wolever et al. (2008) & $\begin{array}{l}\text { Estudo experimental randomizado/ 162 } \\
\text { indivíduos com diabetes tipo 2/ acompanhados } \\
\text { por 01 ano }\end{array}$ & $\begin{array}{l}\text { Em pacientes com DM2 tratados somente com } \\
\text { dieta, que têm controle glicêmico ótimo, a } \\
\text { HbA1c a longo prazo não foi afetada pela } \\
\text { alteração da fonte ou da quantidade de } \\
\text { carboidratos da dieta. Dieta de baixo IG } \\
\text { provocou reduções sustentadas na glicemia e } \\
\text { pode ser preferível para o manejo dietético. }\end{array}$ \\
\hline
\end{tabular}

Fonte: Produzido pelos autores (2021).

Dos vinte e oito artigos reunidos nesta revisão, nove (32\%) investigaram a influência do consumo dessas dietas no controle da DM2. Dentre esses, observou-se que quatro (44,44 \%) das intervenções verificaram efeitos positivos; três (33,33\%) sem efeitos e dois $(22,22 \%)$ efeitos negativos.

O estudo de Meng et al. (2017) observou um melhor controle do DM2, pela diminuição na hemoglobina glicada (HbA1C). Já Hu \& Bazzano (2013), na revisão sistemática, mostraram que a maioria dos ensaios clínicos randomizados publicados concluiu sua que a diminuição de glicemia em jejum, HbA1c, insulina sérica e/ou HOMA foi similar ao observado pelo consumo de dietas isocalóricas com baixo teor de gordura; corroborando com o estudo de Goldstein et al. (2011).

Von et al. (2013) observaram que o consumo de dieta LCHFHP reduziu os valores de HbA1c de forma semelhante ao observado em dietas com baixo teor de gordura e alto teor de carboidrato, sendo que esta também melhorou a resistência à insulina; conforme foi observado no estudo de $\mathrm{Hu} \&$ Bazzano (2013).

Os estudos de Keiko et al. (2014); Wolever et al. (2008); Lawrence et al. (2011); Goldstein et al. (2011) \& Von et al. (2013), não observaram melhora e, em alguns, houve até piora do quadro clínico. Wolever et al. (2008) mostraram que a longo prazo a ingestão de carboidratos de baixo Índice glicêmico seria mais importante do que alteração no tipo ou composição de carboidrato; contrariando os achados de Westman et al. (2008), em que 95,2\% dos indivíduos que consumia uma dieta com redução de carboidratos conseguiram uma diminuição ou eliminação dos medicamentos utilizados para controle do DM2, quando comparado aos que consumiam uma dieta com carboidratos de baixo índice glicêmico (62\%). Já Lawrence et al. (2011) observaram melhora apenas quando a maior fonte de proteína era de origem vegetal, contrapondo Goldstein et al. (2011) ao concluírem que a melhora era similar à dieta convencional.

Dentre os pontos fortes dos estudos analisados, destacam-se o fato de todos serem do tipo experimentais randomizados, com exceção do estudo de coorte prospectivo realizado por Lawrence et al. (2011), bem como tratarem-se de ensaios clínicos, exceto Keiko et al. (2014) \& Alaa et al. (2009), em que a amostras eram compostas por ratos diabéticos. Convém ressaltar os estudos de Westerman et al. (2008) \& Saslow et al. (2014) em que as amostras eram compostas de indivíduos diabéticos sobrepesados ou obesos.

Vários pontos fracos foram verificados nos estudos, tais como: tempo de acompanhamento a curto prazo; pequena amostra de animais ou pessoas; análise e apresentação de apenas registros alimentares, podendo repercutir na estimativa da ingestão alimentar; equações utilizadas para cálculos dos requisitos energéticos; não estratificação por sexo; exclusão dos indivíduos que não utilizavam insulina, não podendo estender resultados para esse público; alguns estudos incluíam sujeitos já diabéticos e pré-diabetes, fator que pode interferir no resultado; consumo de dietas com elevado déficit energético, além da grande variação na distribuição percentual dos macronutrientes; baixa adesão ao consumo da dieta; não seguimento da dieta de forma precisa; e inconstância de manutenção da medicação anti-diabética. 


\section{Consumo de Dieta Low Carb High Fat High Protein na Doença Cardiovascular}

A Tabela 3 mostra uma síntese (autoria, ano de publicação, tipo de intervenção e resultados encontrados) dos estudos que investigaram a influência do consumo da dieta LCHFHP na doença cardiovascular nos participantes do estudo.

Tabela 3 - Síntese dos estudos que investigaram a influência do consumo da dieta Low Carb High Fat High Protein na Doença Cardiovascular.

\begin{tabular}{|c|c|c|}
\hline Autor/Ano & Método & Resultados \\
\hline Tian et al. (2015) & $\begin{array}{l}\text { Estudo experimental randomizado /148 } \\
\text { adultos obesos sem DM2 e DCV/ } \\
\text { acompanhados por } 12 \text { meses }\end{array}$ & $\begin{array}{l}\text { Alterações mais favoráveis com uma dieta com } \downarrow \\
\text { teor de gordura nas concentrações de adiponectina e } \\
\text { ICAM-1. Melhora na inflamação, disfunção } \\
\text { endotelial e disfunção dos adipócitos. }\end{array}$ \\
\hline Megan et al. (2013) & $\begin{array}{l}\text { Estudo experimental randomizado/ } 55 \\
\text { Indivíduos obesos/ acompanhados por } 12 \\
\text { semanas }\end{array}$ & $\begin{array}{l}\text { Melhoras nos marcadores inflamação; HFLC } \downarrow \\
\text { PCR-as em relação ao grupo LFHC, } \uparrow \\
\text { adiponectina. }\end{array}$ \\
\hline Shi et al. (2009) & $\begin{array}{l}\text { Estudo experimental randomizado } \\
\text { realizado com ratos/ acompanhados por } \\
12 \text { semanas }\end{array}$ & $\begin{array}{l}\text { Com } 6 \text { semanas, os ratos no LCHP tinham } \\
\text { significativamente mais ateroma do que ratos no } \\
\text { dieta ocidental (WD). Essa diferença foi mantida } \\
\text { após } 12 \text { semanas nas dietas. Ambos os ratos } \\
\text { alimentados com WD e LCHP desenvolveram } \\
\text { placas maiores que os ratos alimentados com dieta } \\
\text { padrão (SC) }\end{array}$ \\
\hline Peipei et al. (2008) & $\begin{array}{l}\text { Estudo experimental randomizado/ } 12 \\
\text { ratos/ acompanhados por } 02 \text { semanas }\end{array}$ & $\begin{array}{l}\text { Dieta Low carbohydrate (LCD) levou a } \downarrow \text { glicogênio } \\
\text { miocárdico, } \downarrow \text { insulina circulante e } \downarrow \text { ativação } \\
\text { miocárdica de AKT, piora da recuperação da } \\
\text { função, } \uparrow \text { lesão miocárdica após isquemia- } \\
\text { reperfusão no coração isolado; comprometimento } \\
\text { do desempenho do ventrículo esquerdo durante LFI, } \\
\downarrow \text { da recuperação da função após LFI e reperfusão. }\end{array}$ \\
\hline Kostogrys et al. (2012) & $\begin{array}{l}\text { Estudo experimental randomizado/ } 12 \\
\text { ratos fêmeas/ acompanhados por } 10 \\
\text { semanas }\end{array}$ & $\begin{array}{l}\text { Dieta LCHP } \uparrow \text { significativamente a extensão } \\
\text { aterosclerose avançada na raiz da aorta e } \\
\text { braquiocefálica artéria de camundongos e acelera a } \\
\text { vascularização e inflamação. LCHP desenvolveram } \\
\text { aterosclerose mais extensa. }\end{array}$ \\
\hline Bedarida et al. (2014) & $\begin{array}{l}\text { Estudo experimental randomizado/ } 20 \\
\text { ratos jovens e } 40 \text { adultos/ acompanhados } \\
\text { por } 12 \text { semanas }\end{array}$ & $\begin{array}{l}\text { HP-LC leva a equivalentes alterações } \\
\text { cardiovasculares em ratos adultos, como observado } \\
\text { em idade muito avançada, e sublinha o perigo de tal } \\
\text { dieta. Intolerância à glicose e distúrbios lipídicos, } \\
\text { vasculares (especialmente microvasos) e funções } \\
\text { cardíacas, entre outras. Deterioração da função } \\
\text { cardíaca com } \downarrow \text { da fração de encurtamento e fração } \\
\text { de ejeção; } \downarrow \text { distensibilidade aórtica e uma forte } \\
\text { tendência ao } \uparrow \text { da espessura da íntima-média } \\
\text { poderiam ser responsáveis por essas deficiências } \\
\text { cardíacas. Inflamação foi evidenciada pela indução } \\
\text { marcada de citocinas pró-inflamatórias nas artérias, } \\
\text { além do } \uparrow \text { do estresse oxidativo pode estar } \\
\text { envolvido na disfunção endotelial. HP-LC dieta } \\
\text { induz danos metabólicos e vasculares, dependendo } \\
\text { idade e longa duração, mesmo após a interrupção da } \\
\text { dieta. }\end{array}$ \\
\hline
\end{tabular}




\begin{tabular}{|l|l|l|}
\hline Jordi et al. (2014) & $\begin{array}{l}\text { Estudo transversal/ 160 indivíduos com } \\
\text { síndrome metabólica/acompanhados por } \\
03 \text { dias }\end{array}$ & $\begin{array}{l}\downarrow \text { quantidade de carboidratos, mas quantidades } \uparrow \text { de } \\
\text { proteína e gordura, está associada a reatividade } \\
\text { vascular } \downarrow \text { com disfunção endotelial de artéria } \\
\text { pequena em pacientes com SM (diabéticos) e } \uparrow \text { do } \\
\text { risco de doença cardiovascular. }\end{array}$ \\
\hline Pagona et al. (2012) & $\begin{array}{l}\text { Estudo de coorte prospectivo/ 43.369 } \\
\text { mulheres suecas/ acompanhadas por 15,7 } \\
\text { anos }\end{array}$ & $\begin{array}{l}\text { Uma } \downarrow \text { na ingestão de carboidratos e/ou } \uparrow \text { ingestão } \\
\text { de proteínas foram estatisticamente significativas } \\
\text { em associar } \uparrow \text { da incidência de doença } \\
\text { cardiovascular em geral. Dietas com } \downarrow \text { teor de } \\
\text { carboidratos e } \uparrow \text { proteína, usadas regularmente e } \\
\text { sem levar em consideração a natureza dos } \\
\text { carboidratos ou fonte de proteínas, estão associadas } \\
\text { ao } \uparrow \text { do risco de doença cardiovascular. }\end{array}$ \\
\hline Kostogrys et al. (2015) & $\begin{array}{l}\text { Estudo experimental randomizado/ 80 } \\
\text { ratos/ acompanhados por 10 semanas }\end{array}$ & $\begin{array}{l}\text { O efeito pró-aterogênico da dieta LCHP em ratos } \\
\text { está associado à elevação sérica de LDL-c, VLDL, } \\
\text { triacilglicerois e da expressão hepática do SREBP-1 } \\
\text { e inflamação sistêmica. }\end{array}$ \\
\hline
\end{tabular}

Fonte: Produzido pelos autores (2021).

Dos vinte e oito artigos reunidos nesta revisão, dez (35,71\%) investigaram a influência do consumo dessas dietas nas doenças cardiovasculares (DVC). Dentre esses, observou-se que em apenas um estudo (Megan et al., 2013) verificou melhora quanto a esse fator $(10 \%)$.

As pesquisas de Tian et al. (2015); Kostogrys et al. (2012); Bedarida et al. (2014); Jordi et al.(2014) \& Kostogryset et al. (2015) observaram piora nos marcadores inflamatórios, ao contrário do observado por Megan et al. (2013) em que verificaram melhora nos mesmos.

No estudo de Merino et al. (2012), em concordância com os resultados encontrados, onde seus efeitos na função vascular permaneceram negativos ou incertos, foi verificado que este tipo de dieta afetou a reatividade arterial da artéria pequena, visto em pacientes com síndrome metabólica.

Na revisão sistemática realizada por Hu \& Bazzano (2013), foi observado que dietas pobres em carboidratos podem melhorar a função endotelial da carótida, adipocitocinas, citocinas inflamatórias, como a proteína C-reativa e a homocisteína. Também Megan et al. (2013) verificaram efeitos benéficos. Por outro lado, estudo de Ha et al. (2018) mostrou em indivíduos coreanos adultos uma redução do HDL-c com este tipo de dieta; redução essa que, segundo Nicholls e Nelson (2018), está intimamente relacionada ao risco de infarto do miocárdio.

A maioria dos estudos foi do tipo experimental randomizado, com exceção da pesquisa transversal de Jordi et al. (2014) e de coorte prospectiva de Pagona et al. (2012), e realizada com ratos, exceto os estudos de Tian et al. (2015) e Megan et al. (2013), nos quais envolveram adultos obesos.

Dentre os pontos fortes verificados nos estudos, destacam-se a variedade de testes de função cardíaca; determinação de possíveis mecanismos pró- aterogênicos decorrentes do consumo de dietas LCHFHP; pareamento em número de participantes por idade e parâmetros antropométricos.

Dentre os pontos fracos, ressaltam-se a pequena amostra; curto período de tempo; em alguns estudos os dietistas não foram cegados para as hipóteses do estudo; grande parte dos estudos foi realizada em ratos; dietas não isocalóricas; e a ocorrência de desistências, devido às metodologias de análise invasivas (biópsia de tecido adiposo). 
Research, Society and Development, v. 10, n. 16, e282101623575, 2021

(CC BY 4.0) | ISSN 2525-3409 | DOI: http://dx.doi.org/10.33448/rsd-v10i16.23575

\section{Conclusão}

A maioria dos estudos embora demonstrando efeitos positivos do consumo de dieta LCHFHP, ao curto prazo, quanto à perda de peso, redução no percentual de gordura corporal, melhora da sensibilidade à insulina e no controle glicêmico, mostraram que nem sempre esses efeitos foram atribuídos à redução de carboidratos na dieta; e, além desses benefícios não terem sido evidenciados ao longo prazo, observou-se aumento nos níveis séricos dos biomarcadores inflamatórios, da extensão da aterosclerose e na morbidade e mortalidade cardiovascular.

Dessa forma, recomenda-se que mais estudos, principalmente ao longo prazo e com amostragem maior, sejam realizados no sentido de melhor averiguar os benefícios ou riscos decorrentes do consumo dessas dietas, bem como mecanismo de ação, principalmente diante das doenças crônicas não transmissíveis.

\section{Referências}

Al-Khalifa, A., Mathew, T. C., Al-Zaid, N. S., Mathew, E., \& Dashti, H. M. (2009). Therapeutic role of low-carbohydrate ketogenic diet in diabetes. Nutrition, 25(11-12), 1177-1185. https://doi.org/10.1016/j.nut.2009.04.004

American Diabetes Association. (2019). Standards of medical care in diabetes - 2017. Alexandria, Va] American Diabetes Association.

Ballard, K. D., Quann, E. E., Kupchak, B. R., Volk, B. M., Kawiecki, D. M., Fernandez, M. L., Seip, R. L., Maresh, C. M., Kraemer, W. J., \& Volek, J. S. (2013). Dietary carbohydrate restriction improves insulin sensitivity, blood pressure, microvascular function, and cellular adhesion markers in individuals taking statins. Nutrition Research (New York, N.Y.), 33(11), 905-912. https://doi.org/10.1016/j.nutres.2013.07.022

Bedarida, T., Baron, S., Vessieres, E., Vibert, F., Ayer, A., Marchiol-Fournigault, C., Henrion, D., Paul, J.-L., Noble, F., Golmard, J.-L., Beaudeux, J.-L., Cottart, C.-H., \& Nivet-Antoine, V. (2014). High-protein-low-carbohydrate diet: deleterious metabolic and cardiovascular effects depend on age. American Journal of Physiology-Heart and Circulatory Physiology, 307(5), H649-H657. https://doi.org/10.1152/ajpheart.00291.2014

Busetto, L., Marangon, M., \& De Stefano, F. (2011). High-protein low-carbohydrate diets: what is the rationale? Diabetes/Metabolism Research and Reviews, 27(3), 230-232. https://doi.org/10.1002/dmrr.1171

Galletly, C., Moran, L., Noakes, M., Clifton, P., Tomlinson, L., \& Norman, R. (2007). Psychological benefits of a high-protein, low-carbohydrate diet in obese women with polycystic ovary syndrome-A pilot study. Appetite, 49(3), 590-593. https://doi.org/10.1016/j.appet.2007.03.222

Coleman, M. D., \& Nickols-Richardson, S. M. (2005). Urinary ketones reflect serum ketone concentration but do not relate to weight loss in overweight premenopausal women following a low-carbohydrate/high-protein diet. Journal of the American Dietetic Association, 105(4), 608-611. https://doi.org/10.1016/j.jada.2005.01.004

de Luis, D. A., Izaola, O., Aller, R., de la Fuente, B., Bachiller, R., \& Romero, E. (2015). Effects of a high-protein/low carbohydrate versus a standard hypocaloric diet on adipocytokine levels and insulin resistance in obese patients along 9months. Journal of Diabetes and Its Complications, 29(7), 950-954. https://doi.org/10.1016/j.jdiacomp.2015.06.002

Ghroubi, S., Elleuch, H., Chikh, T., Kaffel, N., Abid, M., \& Elleuch, M. H. (2009). Physical training combined with dietary measures in the treatment of adult obesity. A comparison of two protocols. Annals of Physical and Rehabilitation Medicine, 52(5), 394-413. https://doi.org/10.1016/j.rehab.2008.12.017

Goldstein, T., Kark, J. D., Berry, E. M., Adler, B., Ziv, E., \& Raz, I. (2011). The effect of a low carbohydrate energy-unrestricted diet on weight loss in obese type 2 diabetes patients - A randomized controlled trial. E-SPEN, the European E-Journal of Clinical Nutrition and Metabolism, 6(4), e178-e186. https://doi.org/10.1016/j.eclnm.2011.04.003

Ha, K., Joung, H., \& Song, Y. (2018). Low-carbohydrate diet and the risk of metabolic syndrome in Korean adults. Nutrition, Metabolism and Cardiovascular Diseases, 28(11), 1122-1132. https://doi.org/10.1016/j.numecd.2018.06.007

Hu, T., \& Bazzano, L. A. (2014). The low-carbohydrate diet and cardiovascular risk factors: Evidence from epidemiologic studies. Nutrition, Metabolism and Cardiovascular Diseases, 24(4), 337-343. https://doi.org/10.1016/j.numecd.2013.12.008

Green, J. G., Johnson, N. A., Sachinwalla, T., Cunningham, C. W., Thompson, M. W., \& Stannard, S. R. (2010). Low-carbohydrate diet does not affect intramyocellular lipid concentration or insulin sensitivity in lean, physically fit men when protein intake is elevated. Metabolism, 59(11), 1633-1641. https://doi.org/10.1016/j.metabol.2010.03.013

Tay, J., de Bock, M. I., \& Mayer-Davis, E. J. (2019). Low-carbohydrate diets in type 2 diabetes. The Lancet Diabetes \& Endocrinology, 7(5), 331-333. https://doi.org/10.1016/s2213-8587(18)30368-1

Liu, J., \& Lloyd, S. G. (2013). High-fat, low-carbohydrate diet alters myocardial oxidative stress and impairs recovery of cardiac function after ischemia and reperfusion in obese rats. Nutrition Research, 33(4), 311-321. https://doi.org/10.1016/j.nutres.2013.02.005

Merino, J., Kones, R., Ferré, R., Plana, N., Girona, J., Aragonés, G., Ibarretxe, D., Heras, M., \& Masana, L. (2013). Low-carbohydrate, high-protein, high-fat diet alters small peripheral artery reactivity in metabolic syndrome patients. Clinica E Investigacion En Arteriosclerosis: Publicacion Oficial de La Sociedad Espanola de Arteriosclerosis, 26(2), 58-65. https://doi.org/10.1016/j.arteri.2013.11.004 
Hall, Kevin D., Bemis, T., Brychta, R., Chen, Kong Y., Courville, A., Crayner, Emma J., Goodwin, S., Guo, J., Howard, L., Knuth, Nicolas D., Miller, Bernard V., Prado, Carla M., Siervo, M., Skarulis, Monica C., Walter, M., Walter, Peter J., \& Yannai, L. (2015). Calorie for Calorie, Dietary Fat Restriction Results in More Body Fat Loss than Carbohydrate Restriction in People with Obesity. Cell Metabolism, 22(3), 427-436. https://doi.org/10.1016/j.cmet.2015.07.021

Handa, K., Inukai, K., Onuma, H., Kudo, A., Nakagawa, F., Tsugawa, K., Kitahara, A., Moriya, R., Takahashi, K., Sumitani, Y., Hosaka, T., Kawakami, H., Oyadomari, S., \& Ishida, H. (2014). Long-Term Low Carbohydrate Diet Leads to Deleterious Metabolic Manifestations in Diabetic Mice. PLoS ONE, 9(8). https://doi.org/10.1371/journal.pone.0104948

Kim, J.-Y., Yang, Y.-H., Kim, C.-N., Lee, C.-E., \& Kim, K.-I. (2008). Effects of Very-Low-Carbohydrate (Horsemeat- or Beef-Based) Diets and Restricted Feeding on Weight Gain, Feed and Energy Efficiency, as well as Serum Levels of Cholesterol, Triacylglycerol, Glucose, Insulin and Ketone Bodies in Adult Rats. Annals of Nutrition and Metabolism, 53(3-4), 260-267. https://doi.org/10.1159/000189129

Kostogrys, R. B., Franczyk-Żarów, M., Maślak, E., Gajda, M., Mateuszuk, Ł., Jackson, C. L., \& Chłopicki, S. (2012). Low carbohydrate, high protein diet promotes atherosclerosis in apolipoprotein E/low-density lipoprotein receptor double knockout mice (apoE/LDLR-/-). Atherosclerosis, 223(2), 327-331. https://doi.org/10.1016/j.atherosclerosis.2012.05.024

Leonard, E. A., \& Marshall, R. J. (2018). Cardiovascular Disease in Women. Primary Care, 45(1), 131-141. https://doi.org/10.1016/j.pop.2017.10.004

de Koning, L., Fung, T. T., Liao, X., Chiuve, S. E., Rimm, E. B., Willett, W. C., Spiegelman, D., \& Hu, F. B. (2011). Low-carbohydrate diet scores and risk of type 2 diabetes in men. The American Journal of Clinical Nutrition, 93(4), 844-850. https://doi.org/10.3945/ajcn.110.004333

Ruth, M. R., Port, A. M., Shah, M., Bourland, A. C., Istfan, N. W., Nelson, K. P., Gokce, N., \& Apovian, C. M. (2013). Consuming a hypocaloric high fat low carbohydrate diet for 12 weeks lowers C-reactive protein, and raises serum adiponectin and high density lipoprotein-cholesterol in obese subjects. Metabolism, 62(12), 1779-1787. https://doi.org/10.1016/j.metabol.2013.07.006

Meng, Y., Bai, H., Wang, S., Li, Z., Wang, Q., \& Chen, L. (2017). Efficacy of low carbohydrate diet for type 2 diabetes mellitus management: A systematic review and meta-analysis of randomized controlled trials. Diabetes Research and Clinical Practice, 131, 124-131. https://doi.org/10.1016/j.diabres.2017.07.006

Krebs, N. F., Gao, D., Gralla, J., Collins, J. S., \& Johnson, S. L. (2010). Efficacy and Safety of a High Protein, Low Carbohydrate Diet for Weight Loss in Severely Obese Adolescents. The Journal of Pediatrics, 157(2), 252-258. https://doi.org/10.1016/j.jpeds.2010.02.010

Nicholls, S. J., \& Nelson, A. J. (2019). HDL and cardiovascular disease. Pathology, 51(2), 142-147. https://doi.org/10.1016/j.pathol.2018.10.017

Nilsson, L. M., Winkvist, A., Eliasson, M., Jansson, J-H., Hallmans, G., Johansson, I., Lindahl, B., Lenner, P., \& Van Guelpen, B. (2012). Low-carbohydrate, high-protein score and mortality in a northern Swedish population-based cohort. European Journal of Clinical Nutrition, 66(6), 694-700. https://doi.org/10.1038/ejen.2012.9

Lagiou, P., Sandin, S., Lof, M., Trichopoulos, D., Adami, H.-O. \& Weiderpass, E. (2012). Low carbohydrate-high protein diet and incidence of cardiovascular diseases in Swedish women: prospective cohort study. BMJ, 344(jun26 3), e4026-e4026. https://doi.org/10.1136/bmj.e4026

Wang, P., Tate, J. M., \& Lloyd, S. G. (2008). Low carbohydrate diet decreases myocardial insulin signaling and increases susceptibility to myocardial ischemia. Life Sciences, 83(25-26), 836-844. https://doi.org/10.1016/j.lfs.2008.09.024

Rho, J. M., \& Sankar, R. (2008). The ketogenic diet in a pill: Is this possible? Epilepsia, 49, 127-133. https://doi.org/10.1111/j.1528-1167.2008.01857.x

Samaha, F. F., Iqbal, N., Seshadri, P., Chicano, K. L., Daily, D. A., McGrory, J., Williams, T., Williams, M., Gracely, E. J., \& Stern, L. (2003). A LowCarbohydrate as Compared with a Low-Fat Diet in Severe Obesity. New England Journal of Medicine, 348(21), 2074-2081. https://doi.org/10.1056/nejmoa022637

Saslow, L. R., Kim, S., Daubenmier, J. J., Moskowitz, J. T., Phinney, S. D., Goldman, V., Murphy, E. J., Cox, R. M., Moran, P., \& Hecht, F. M. (2014). A Randomized Pilot Trial of a Moderate Carbohydrate Diet Compared to a Very Low Carbohydrate Diet in Overweight or Obese Individuals with Type 2 Diabetes Mellitus or Prediabetes. PLoS ONE, 9(4), e91027. https://doi.org/10.1371/journal.pone.0091027

Sociedade Brasileira De Diabetes. (2017). Diretrizes da Sociedade Brasileira de Diabetes 2017-2018 (CLANNAD, Ed.) [Review of Diretrizes da Sociedade Brasileira de Diabetes 2017-2018]. (Original work published 2018)

Sansbury, B. E., \& Hill, B. G. (2014). Regulation of obesity and insulin resistance by nitric oxide. Free Radical Biology and Medicine, 73, 383-399. https://doi.org/10.1016/j.freeradbiomed.2014.05.016

Soenen, S., Bonomi, A. G., Lemmens, S. G. T., Scholte, J., Thijssen, M. A. M. A., van Berkum, F., \& Westerterp-Plantenga, M. S. (2012). Relatively highprotein or "low-carb" energy-restricted diets for body weight loss and body weight maintenance? Physiology \& Behavior, 107(3), 374-380. https://doi.org/10.1016/j.physbeh.2012.08.004

Shi, Y. F.; Eric, R. H.; Joanna, W.; Christopher, J. S.; Jennifer, J. M.; Kathryn, J. M.; Robert, E. G.; Anthony, R. (2012). Vascular effects of a lowcarbohydrate high-protein diet [Review of Vascular effects of a low-carbohydrate high-protein diet]. PNAS, 36(116).

Stocks, T., Taylor, M. A., Ängquist, L., MacDonald, I. A., Arner, P., Holst, C., Oppert, J.-M., Martinez, J. Alfredo., Rössner, S., Polak, J., Langin, D., Saris, W. H. M., Astrup, A., \& Sørensen, T. I. A. (2013). Change in Proportional Protein Intake in a 10-Week Energy-Restricted Low- or High-Fat Diet, in Relation to Changes in Body Size and Metabolic Factors. Obesity Facts, 6(3), 217-227. https://doi.org/10.1159/000351726

Hu, T., Yao, L., Reynolds, K., Whelton, P., Niu, T., Li, S., He, J., \& Bazzano, L. (2015). The Effects of a Low-Carbohydrate Diet vs. a Low-Fat Diet on Novel Cardiovascular Risk Factors: A Randomized Controlled Trial. Nutrients, 7(9), 7978-7994. https://doi.org/10.3390/nu7095377 
Research, Society and Development, v. 10, n. 16, e282101623575, 2021

(CC BY 4.0) | ISSN 2525-3409 | DOI: http://dx.doi.org/10.33448/rsd-v10i16.23575

von Bibra, H., Wulf, G., St John Sutton, M., Pfützner, A., Schuster, T., \& Heilmeyer, P. (2014). Low-carbohydrate/high-protein diet improves diastolic cardiac function and the metabolic syndrome in overweight-obese patients with type 2 diabetes. IJC Metabolic \& Endocrine, 2, 11-18. https://doi.org/10.1016/j.ijcme.2013.12.001

Westman, E. C., Yancy, W. S., Mavropoulos, J. C., Marquart, M., \& McDuffie, J. R. (2008). The effect of a low-carbohydrate, ketogenic diet versus a lowglycemic index diet on glycemic control in type 2 diabetes mellitus. Nutrition \& Metabolism, 5(1). https://doi.org/10.1186/1743-7075-5-36

Faludi, A. A. et al. (2017). Atualização da diretriz brasileira de dislipidemias e prevenção da aterosclerose (Arquivos Brasileiros de Cardiologia, Ed.; 2nd ed., Vol. 109) [Review of Atualização da diretriz brasileira de dislipidemias e prevenção da aterosclerose].

Wolever, T. M., Gibbs, A. L., Mehling, C., Chiasson, J.-L., Connelly, P. W., Josse, R. G., Leiter, L. A., Maheux, P., Rabasa-Lhoret, R., Rodger, N. W., \& Ryan, E. A. (2008). The Canadian Trial of Carbohydrates in Diabetes (CCD), a 1-y controlled trial of low-glycemic-index dietary carbohydrate in type 2 diabetes: no effect on glycated hemoglobin but reduction in C-reactive protein. The American Journal of Clinical Nutrition, 87(1), 114-125. https://doi.org/10.1093/ajen/87.1.114 